\title{
THEORY OF THE GAIN CHARACTERISTICS OF InGaN/AIGaN QD LASERS
}

\author{
A.D. Andreev * and E.P. O’Reilly ** \\ * A.F. Ioffe Physico-Technical Institute of Russian Academy of Sciences, \\ Polytechnicheskaya 26, St.-Petersburg 194021, Russia; E-mail: A.Andreev@surrey.ac.uk \\ ** Department of Physics, University of Surrey, Guildford GU2 5XH, UK
}

\section{ABSTRACT}

We present a theoretical analysis of the gain characteristics of InGaN/AlGaN quantum dot (QD) lasers. We calculate the elastic strain distribution caused by the lattice mismatch between the QD and the barrier using an original method which takes into account the hexagonal symmetry of the structure's elastic properties. The method is based on an analytical derivation of the Fourier transform of the strain tensor. The proposed approach is combined with a plane-wave expansion method to calculate the carrier spectrum and wave functions. The many-body gain of a laser containing a periodic array of QDs is calculated using the Padé approximation. We show that band gap reduction and the Coulomb enhancement of the interband transition probability can significantly modify the gain spectrum in InGaN/AlGaN QD lasers.

\section{INTRODUCTION}

Wide-band gap semiconductors are attracting considerable attention because of their potential as visible light emitting diodes [1]. At the same time, advances in growth technology has stimulated active investigations in the field of zero dimensional quantum dot (QD) structures. The first GaN-based structures with self-organized QDs have been fabricated recently [2-4]. Lasers based on self-organized QDs have potential advantages over conventional quantum well lasers because of their atomic-like density of states. For wide-band gap heterostructures additional benefits may arise from using QD structures instead of quantum well ones. Firstly, strain relaxation in QD structures is easier and therefore defect-free fabrication of strongly latticemismatched heterostructures may be possible. Secondly, because of the carrier localization, the role of many-body effects may increase, resulting in a stronger gain enhancement due to Coulomb interactions. Moreover, recent publications [5,6] demonstrate that light emission from InGaN quantum well lasers may originate from dot-like localized states formed because of indium composition fluctuations. It is therefore timely to undertake a theoretical investigation of the gain characteristics of GaN-based QD structures.

We present here a theoretical study of the elastic strain distribution, carrier spectrum and many-body gain in structures consisting of a periodic array of InGaN QDs embedded in an AlGaN matrix. Employing the Green's function and Fourier transform techniques we find the 3D strain distribution in the QDs. To calculate the carrier spectrum and wave functions we use the planewave expansion method. This method allows us to take account of the strain-induced modification of the band structure using analytical expressions for the Fourier transform of the strain tensor. We thus avoid explicit calculation of the strain distribution. The calculated carrier spectrum and wave functions are used as input for gain calculations including many-body effects. We demonstrate that band gap reduction and Coulomb enhancement of the interband transition probability significantly modify the gain spectrum.

For ease of calculation we use a model structure which assumes that the QDs all have the same shape and size, and form a periodic structure with periods $d_{\|}$and $d_{z}$ in the lateral and growth directions respectively. 


\section{CALCULATION OF STRAIN DISTRIBUTION AND CARRIER SPECTRUM}

The calculation of the spatial strain distribution in a QD structure requires solving a 3D problem in elasticity theory for a generally non-trivial quantum dot shape. This is often achieved by using finite-difference methods or atomistic techniques [7,8]. These methods require considerable computational effort. A simple method to calculate the strain field in semiconductor structures with QDs of arbitrary shape was presented in Ref. [9] for the case when the elastic properties are assumed to be isotropic and the elastic constants of the QD and matrix materials are equal. The effects of anisotropic elastic constants on the strain distribution were included later in [10] for cubic crystals. We briefly describe here the generalization of this approach for hexagonal crystals (details are given in [11]).

Consider a QD of arbitrary shape formed by embedding one kind of elastic material into a second elastic material with hexagonal symmetry. The displacement vector due to the lattice mismatch between the QD and matrix is expressed through the Green's tensor $\mathrm{G}_{\mathrm{in}}(\mathbf{r}, \mathbf{r}$ ') of the elastic equation:

$$
u_{i}=u_{i}^{(0)}+\int_{\Omega^{\prime}} G_{i n}\left(r, r^{\prime}\right) \lambda_{n k p r}^{Q D} e_{p r}^{(0)} d S_{k}^{\prime} .
$$

Here we use the usual rule for summation over 1,2,3 (i.e. $\mathrm{x}, \mathrm{y}, \mathrm{z}$ ) for repeating indices; $\lambda_{n k p r}^{Q D D}$ is the elastic tensor of the QD material, $u_{i}^{(0)}$ is the initial displacement associated with the strain tensor $e_{p r}^{(0)}=\varepsilon_{a} \delta_{p r}+\left(\varepsilon_{a}-\varepsilon_{c}\right) \delta_{p 3} \delta_{r 3}$, where $\varepsilon_{a}=\left(a^{M}-a^{\beta D}\right) / a^{\beta D}$ and $\varepsilon_{c}=\left(c^{M}-c^{Q D}\right) / c^{\beta D}$ is the lattice mismatch. Integration in (1) is carried out over the QD surface. The Green's tensor is the solution of the equation:

$$
\frac{\partial}{\partial x_{k}} \lambda_{i k l m}(r) \frac{\partial}{\partial x_{m}} G_{l n}\left(r, r^{\prime}\right)=-\delta\left(r-r^{\prime}\right) \delta_{i n} .
$$

Using a Fourier transform technique we solve Eq. (2) and find the Fourier transform of the strain tensor $e_{i j}$ which corresponds to the displacement $u_{i}$ in the structure with a single QD. When the elastic constants of the QD and matrix materials are equal, the solution of eq. (2) has the form [11]:

$$
\tilde{e}_{i j}^{s}(\xi)=\tilde{\chi}_{Q D}(\xi)\left\{e_{p r}^{(0)}+\frac{(R P-S I) \xi_{i} \xi_{j}+(-R Q+S F) \xi_{3}\left(\xi_{i} \delta_{j 3}+\xi_{i} \delta_{j 3}\right) / 2}{I Q-F P}\right\},
$$

where

$$
\begin{array}{lrr}
Q= & \left(C_{13}+2 C_{44}-C_{11}\right) \xi^{2}+\left(C_{33}-2 C_{13}-4 C_{44}+C_{11}\right) \xi_{3}^{2} ; & I=\left(C_{13}+C_{44}\right) \xi_{3}^{2} ; \\
P=\left(C_{33}-2 C_{44}-C_{13}\right) \xi_{3}^{2}+C_{44} \xi^{2} ; & R=\left(C_{11}+C_{12}+C_{13}\right) \varepsilon_{a}+C_{13}\left(\varepsilon_{c}-\varepsilon_{a}\right) ; \\
F=C_{11} \xi^{2}+\left(C_{13}+2 C_{44}-C_{11}\right) \xi_{3}^{2} ; & S=\left(C_{33}+C_{13}-C_{12}-C_{11}\right) \varepsilon_{a}+\left(C_{33}-C_{13}\right)\left(\varepsilon_{c}-\varepsilon_{a}\right) .
\end{array}
$$

Here $C_{i j}$ denotes the elastic constants, $\xi_{i}$ are the components of the vector in the inverse space. In Eq. (3) $\tilde{\chi}_{Q D}$ denotes the Fourier transform of the QD characteristic function $\chi_{Q D}(\mathbf{r})$, which is equal to unity inside the QD and zero outside. The strain tensor of the periodic array of QDs is then expressed through the Fourier series:

$$
e_{i j}(\mathbf{r})=\frac{(2 \pi)^{3}}{d_{1} d_{2} d_{3}} \sum_{\mathbf{n}} \tilde{e}_{i j}^{s}\left(\xi_{\mathbf{n}}\right) \exp \left(i \mathbf{k}_{\mathbf{n}} \cdot \mathbf{r}\right),
$$

where $\xi_{\mathrm{n}}=2 \pi\left(n_{1} / d_{1}, n_{2} / d_{2}, n_{3} / d_{3}\right), d_{i}$ is the period in the $i$-th direction; and the summation is carried out over all values of $n_{i}$ except the case when $n_{1}=n_{2}=n_{3}=0$ [10].

Equations (3) and (4) give an analytical expression for the strain tensor in a periodic array of QDs of arbitrary shape. It is worth noting that the QD shape enters the expressions only through the form of the Fourier transform of the characteristic function $\chi_{Q D}(\mathbf{r})$, which can be found analytically for most dot shapes considered to date [10]. 
Figures 1 and 2 show the variation of $e_{i j}(\boldsymbol{r})$ through a cylindrical QD and a pyramidal QD respectively. The pyramid has sharp edges along the (001) and (111) directions; therefore near the QD boundary the strain tensor components vary rather steeply.

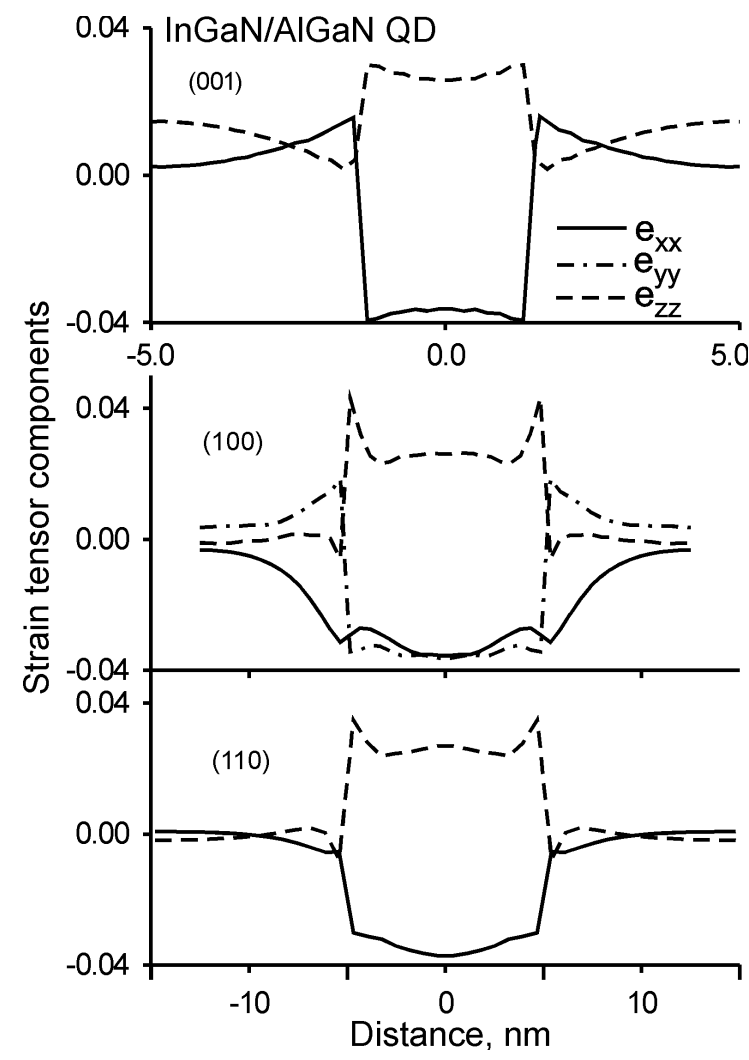

Fig.1 Strain tensor dependence on space position for a cylindrical $\mathrm{In}_{0.5} \mathrm{Ga}_{0.5} \mathrm{~N} /$ $\mathrm{Al}_{0.1} \mathrm{Ga}_{0.9} \mathrm{~N}$ QD. The QD diameter is $10 \mathrm{~nm}$, height is $3 \mathrm{~nm}$, lateral period is $25 \mathrm{~nm}$, and period along $\mathrm{z}$ is $10 \mathrm{~nm}$. Material parameters are from Refs.[12,13].

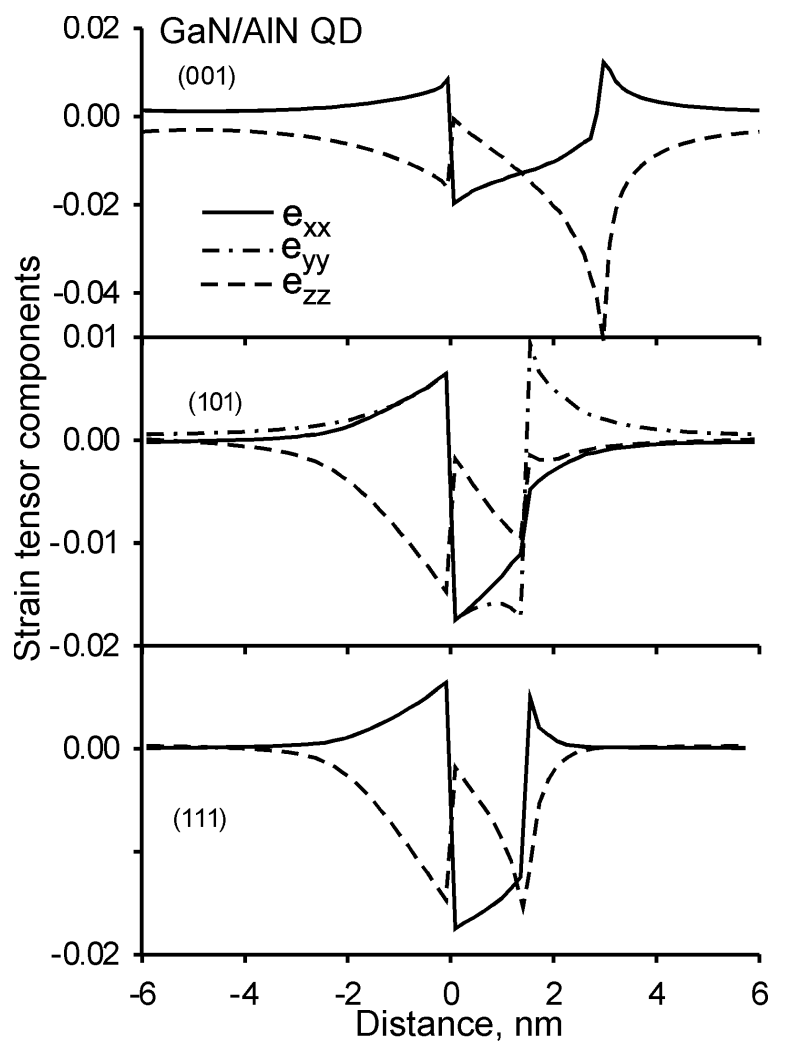

Fig.2 Strain tensor dependence on space position along three different directions for a GaN/AIN pyramidal QD. The QD base is $6 \times 6 \mathrm{~nm}$, the height is $3 \mathrm{~nm}$, lateral period $\mathrm{d}_{/ /}$ $=18 \mathrm{~nm}$, and period along $\mathrm{z}, \mathrm{d}_{\mathrm{z}}=12 \mathrm{~nm}$. Material parameters are from Refs.[12,13].

The multiband envelope function approximation is commonly used to calculate the carrier spectrum in semiconductor quantum structures. This approach has proved to be a convenient and reliable tool to describe quantum size effects in quantum wells (QWs) as well as in QD structures. The coupling between the conduction and valence bands in wide-band gap GaN-based structures is relatively small over the energy range of interest. Therefore we used a simple one-band model with parabolic dispersion for the conduction band; the strain-induced shift of the band-edge is given by $a_{c} e_{i i}(r)$. To describe the valence band states we used a $6 x 6$ Hamiltonian including strainrelated deformation potentials [14,15], but ignoring the strain-induced piezoelectric fields. These fields are larger here than in III-V zinc-blende crystals, but can still be omitted because of the small dot sizes considered below. Because the spin-orbit constants in both GaN and InN are only of order a few meV, we set the spin-orbit interactions to zero, so that the 6x6 Hamiltonian is then decomposed into two independent 3x3 matrices. In contrast to GaAs/InAs-based QD structures, the effect of strain on the valence band edge potential profile is several times smaller in GaNbased QDs. Figure 3 shows the variation of the $\mathrm{HH}, \mathrm{LH}$ and $\mathrm{CH}$ valence band edges along the $z$ and $x$ axes of the cylindrical dot, including the strain-induced splittings. In spite of the relatively large strain (around 4\%), the splitting between the $(\mathrm{H}, \mathrm{L}) \mathrm{H}$ and $\mathrm{CH}$ bands is only around 150 $\mathrm{meV}$, compared to several hundreds of meV for similar GaAs/InAs-based structures. The HH and 
LH practically do not split inside the QD, although a small splitting is found outside. Thus the strain in a cylindrical QD does not split the degeneracy of the HH and LH bands, although it does separate off the $\mathrm{CH}$ band, because of its $z$-like character.

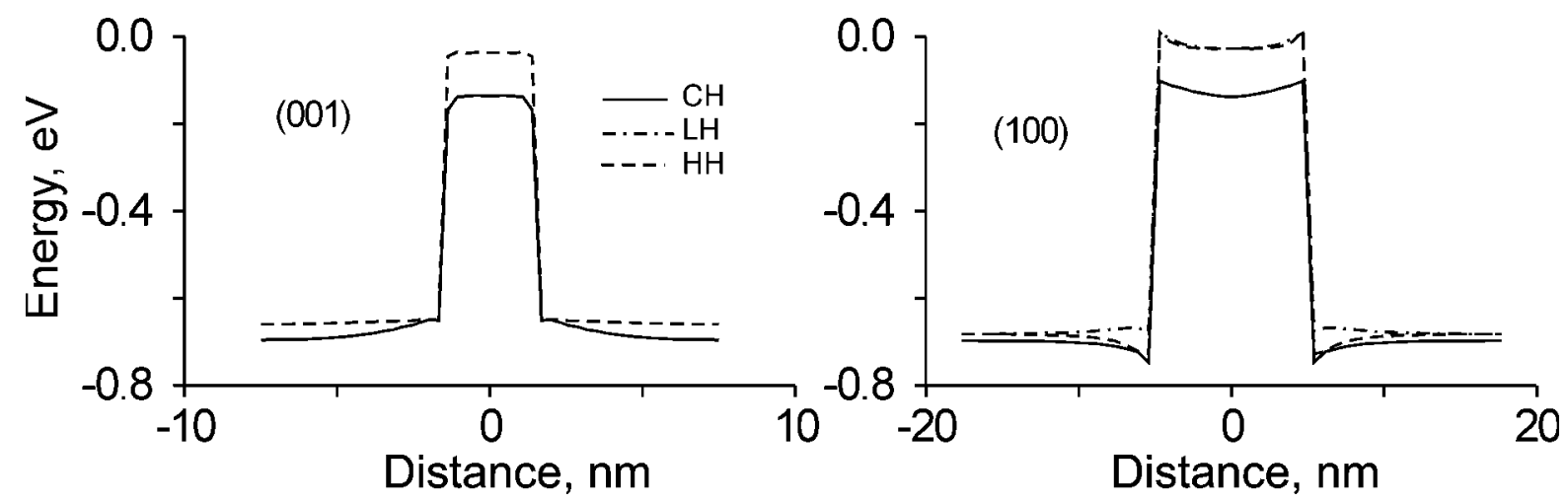

Figure 3. Spatial variation of the valence band edges, including strain-induced splittings. The structure parameters are the same as in Fig.1; the deformation potentials are assumed equal in the QD and matrix, with $D_{1}=0.7 \mathrm{eV}, D_{2}=2.1 \mathrm{eV}, D_{5}=-0.7 \mathrm{eV}$; the barrier height ratio (without strain) is taken as $\mathrm{E}_{\mathrm{v}} / \mathrm{E}_{\mathrm{c}}=7 / 3$ [16].

To calculate the carrier spectrum and wave functions we use a plane-wave expansion method. The idea of this method is to describe the carrier states as a linear combination of a suitably chosen set of bulk states associated with a periodic array of bulk wave vectors. The effective Hamiltonian is represented in the form

$$
\hat{\mathbf{H}}=\hat{\mathbf{H}}_{0}+\hat{\mathbf{V}}
$$

where the 'perturbation' $\hat{\mathbf{V}}$ describes the difference between the potential in a unit cell of the periodic dot array, and the potential in the bulk Hamiltonian $\hat{\mathbf{H}}_{0}$ used to generate the basis states $|\mathbf{k}, \alpha\rangle$, where $\mathbf{k}$ is the momentum and $\alpha$ denote the type of the bulk carrier state, i.e. HH, LH or $\mathrm{CH}$. The operator matrix $\hat{\mathbf{V}}$ in Eq.(5) should be obtained from the bulk-like Hamiltonian by making the substitution $k_{i} \rightarrow-i \partial / \partial_{i}$, to take account of the spatial dependence of the band parameters. Details of the interface boundary conditions are included by an appropriate application of the differential operators at each interface. The solution of the initial Schroedinger equation is found by expanding in a series with respect to the plane waves $|\mathbf{k}, \alpha\rangle \propto \exp (\mathbf{i k . r})$. The coefficients of this series and the energies of the carrier states in an array of QDs are found numerically as the eigenvectors and eigenvalues of a matrix $\mathbf{A}$, involving matrix elements $\mathrm{V}_{\mathbf{k} \alpha, \mathbf{k}^{\prime} \alpha^{\prime}}=\left\langle\mathbf{k}^{\prime} \alpha^{\prime}|\hat{\mathbf{V}}| \mathbf{k}, \alpha\right\rangle$, which are the Fourier transforms of the matrix $\hat{\mathbf{V}}$. The matrix elements $\mathrm{V}_{\mathbf{k} \alpha, \mathbf{k}^{\prime} \alpha}$, depend linearly on the strain components. Therefore the Fourier transform of $\hat{\mathbf{V}}$ is expressed through the Fourier transform of the strain tensor and the QD characteristic function $\chi$ ${ }_{Q D}(\mathbf{r})$. This means that using a plane-wave expansion method in conjunction with the Green's function approach for strain calculations, there is no need to calculate the full spatial strain distribution, unlike in other techniques. This simple trick considerably reduces the computation time to set up calculations and makes the plane-wave method very effective for the further study of QD optical properties and modeling of QD devices. We also note that the number of bulk states ("plane waves") which must be included to obtain a given level of accuracy is reduced in periodic structures with partly coupled QDs.

Figure 4 shows the calculated electron and hole energy levels for InGaN/AlGaN QDs. As the In content increases, the barrier heights become larger both for electrons and holes in the QD, with the energy levels then also shifting further away from the barrier band edges. 

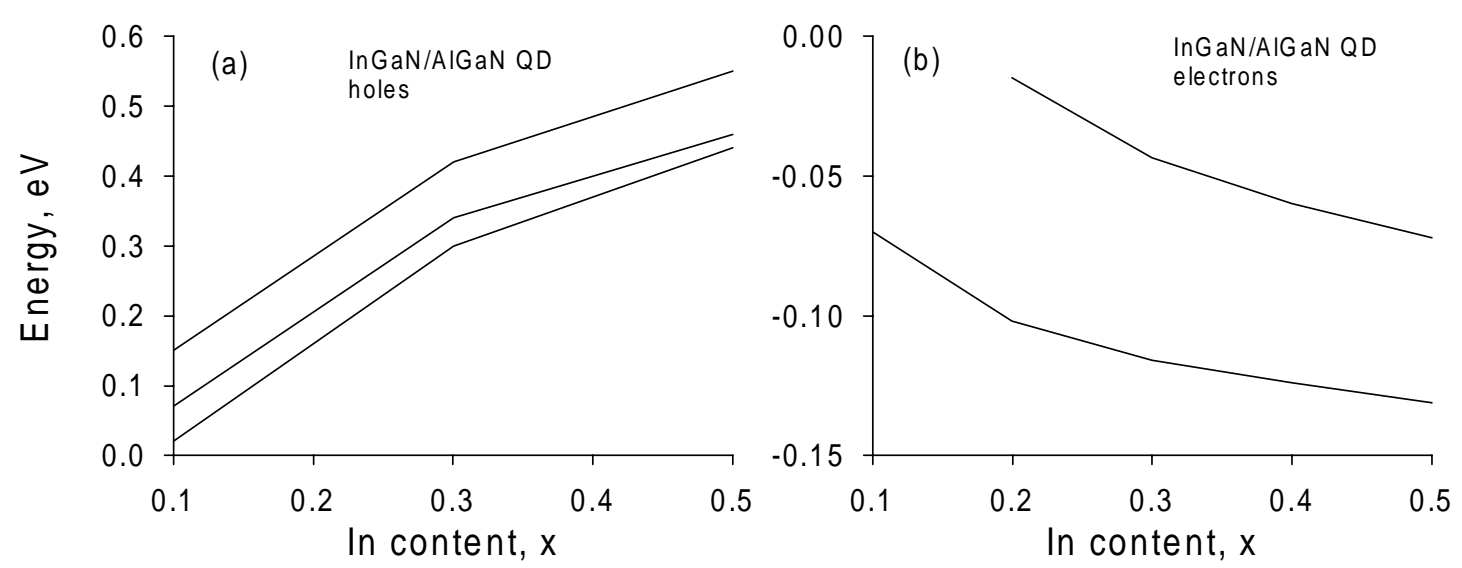

Figure 4. Dependence of the hole and electron energy levels on In content in an $\operatorname{In}_{x} \mathrm{Ga}_{1-\mathrm{x}} \mathrm{N} /$ $\mathrm{Al}_{0.1} \mathrm{G}_{0.9} \mathrm{~N}$ QD array. The energy is measured form the barrier valence (a) or conduction (b) band edge. The QD is cylindrical with a diameter of $5 \mathrm{~nm}$ and height of $2 \mathrm{~nm}$; the periods are $\mathrm{d}_{\|}=10 \mathrm{~nm}$ and $\mathrm{d}_{\mathrm{z}}=5 \mathrm{~nm}$. Valence band parameters are from [17], electron effective masses from [18], and the band offset ratio for unstrained materials is taken as $\mathrm{E}_{\mathrm{v}} / \mathrm{E}_{\mathrm{c}}=7 / 3$ [16].

\section{GAIN MODELING}

Many-body effects $[19,20]$ such as Coulomb enhancement of the interband transition probability and band gap renormalization are important for gain modeling especially for wide band-gap semiconductors [21], because of the high exciton binding energies in these materials. We have used the Padé approximation to model the gain associated with a periodic array of QDs. We considered a structure where the dots are relatively close (the characteristic distance between the dots is comparable to the QD size), so that the dots are partially coupled and form a 3D QD superlattice [22]. We assumed therefore that the Coulomb interaction between the carriers is 3Dlike and used the unscreened Coulomb potential, with $V(q)=4 \pi e^{2} /\left(\varepsilon_{0} q^{2}\right)$. We use a Lorentzian lineshape function to take account of broadening effects. This gives a reasonable description of the higher energy side of the gain spectra and of the peak gain value, but is less reliable on the lower energy side.

Figure 5 shows the calculated gain spectra for an InGaN/AlGaN QD laser. For comparison, the gain spectra calculated without Coulomb enhancement are also shown. We see from Fig.5 that the Coulomb enhancement strongly affects the peak gain value. In particular, at $\mathrm{N}_{\mathrm{e}}=8 \times 10^{18} \mathrm{~cm}^{-3}$

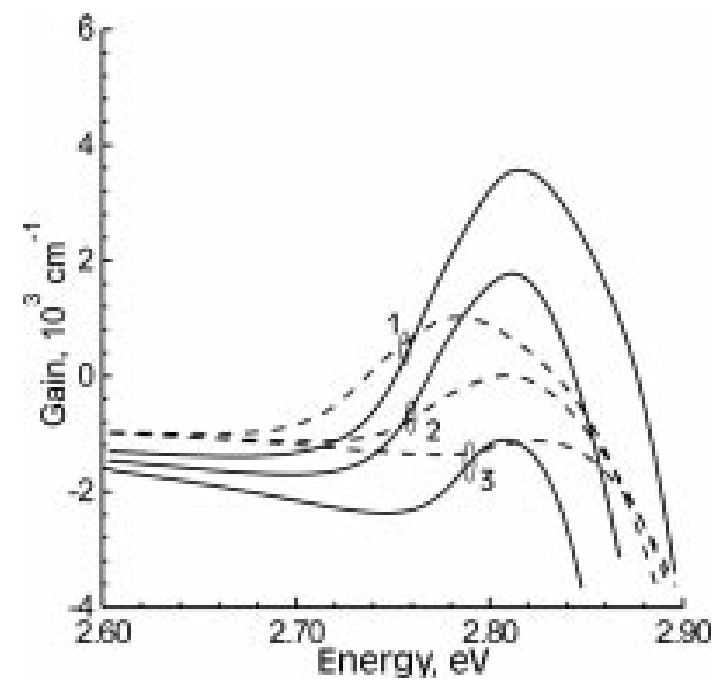

Figure 5. Calculated gain spectra for an $\mathrm{In}_{0.5} \mathrm{Ga}_{0.5} \mathrm{~N} / \mathrm{Al}_{0.1} \mathrm{Ga}_{0.9} \mathrm{~N}$ QD structure at $\mathrm{T}=300 \mathrm{~K}$. Curves 3, 2 and 1 correspond to carrier densities $\left(\mathrm{cm}^{-3}\right)$ of $4 \times 10^{18}$, $6 \times 10^{18}$ and $8 \times 10^{18}$, respectively. Dashed curves are results without Coulomb enhancement. The QDs are disk-like; the diameter and height are $5 \mathrm{~nm}$ and $2 \mathrm{~nm}$ respectively. The QD array periods are $\mathrm{d}_{\|}=7.5 \mathrm{~nm}$ and $\mathrm{d}_{\mathrm{z}}=4 \mathrm{~nm}$. 
the peak gain increases by a factor of 3.5 when the Coulomb enhancement is taken into account (see Fig.5). Thus we demonstrate that including many-body effects strongly modifies the gain spectrum of a laser based on a periodic array of InGaN QDs.

\section{CONCLUSIONS}

In conclusion, we have presented a simple technique to calculate the strain distribution due to a quantum dot embedded in a host crystal with hexagonal symmetry. We have used the technique to calculate the strain distribution associated with cylindrical and pyramidal (In)GaN quantum dots embedded in an AlGaN matrix. Our results for the cylindrical dot were then used as input to calculate the electron and hole confined state energies in a periodic array of cylindrical $\operatorname{In}_{0.5} \mathrm{Ga}_{0.5} \mathrm{~N}$ quantum dots embedded in a $\mathrm{Al}_{0.1} \mathrm{Ga}_{0.9} \mathrm{~N}$ matrix, and these were used to determine the material gain as a function of carrier density, both including and ignoring Coulomb interaction effects. We show that the Coulomb interaction considerably enhances the peak gain at a fixed carrier density, and conclude that InGaN quantum dots should provide a viable gain medium to achieve laser emission in the visible and near-UV spectral regions.

\section{ACKNOWLEDGEMENT}

This work was partially supported by the European Union BRITE-EURAM "RAINBOW" project.

\section{REFERENCES}

1. S. Nakamura, G. Fasol, The Blue Laser Diode: GaN Based Light Emitters and Lasers (Springer-Verlag, Berlin, 1997)

2. F. Widmann, B. Daudin, G. Feuillet, et.al., J. of Appl. Phys, , 83, 7618 (1998)

3. B. Daudin, F. Widmann, G. Feuillet, et.al., Phys. Rev. B, 56, R7069 (1997)

4. H. Hirayama, S. Tanaka, P. Ramvall, Y. Aoyagi, Appl. Phys. Lett., 72, 1736 (1998)

5. J.S. Im, S. Heppel, H. Kollmer, et.al., J. of Grystal Growth, 190, 597 (1998)

6. Y. Narukawa, Y. Kawakami, M. Funato, et.al., Appl. Phys. Lett., 70, 981 (1997)

7. M. Grundman, O. Stier, D. Bimberg, Phys. Rev. B, 52, 11969 (1995)

8. A. Cusack, P. R. Briddon, M. Jaros, Phys. Rev. B, 54, R2300 (1996)

9. J.R. Downes, D.A. Faux, E.P. O'Reilly, J. Appl. Phys., 81, (10), 6700 (1997)

10. A.D. Andreev, J.R. Downes, D.A. Faux, E.P.O'Reilly, subm. to J. Appl.Phys.

11. A.D Andreev, E.P.O'Reilly, to be subm. to Phys.Rev. B.

12. A.F. Wright, J. Appl. Phys., 82, 2833 (1997)

13. T.L. Tansley, E.M. Goldys, H. Godlevski, et.al., in GaN and Related Materials, edited by S.J. Pearton (Gordon and Breach Science Publish., New York, 1997), p.268

14. G.L. Bir, G.E. Pikus, Symmetry and Strain-Induced Effects in Semiconductors (Wiley, New York, 1972)

15. S.L. Chuang, C.S. Chang, Phys. Rev. B, 54, 2491 (1996)

16. G. Martin, A. Botchkarev, A. Rockett, H. Morcos, Appl. Phys. Lett., 68, 2541 (1996)

17. T.C. Yeo, T.C. Chong, M.F. Li, J. Appl.Phys., 83, 1429 (1998)

18. T.C. Chong, Y.C. Yeo, M.F. Li, W.J. Fan, MRS Proc. vol. 482 (1997)

19. H. Haug, S.W. Koch, Phys. Rev. A, 39, 1887 (1989)

20. W.W. Chow, S.T. Koch, M. Sargent III, IEEE J. of Quantum Electronics, 26, 1052 (1990)

21. P.Rees, C. Cooper, P.Blood, et.al., Electron. Lett., 31, 1149 (1995)

22. A.D. Andreev, in In-plane Semiconductor Lasers: from Ultraviolet to Mid-infrared, SPIE Proc., 3284, 151 (1998) 